\title{
Approximate Inertial Manifold for a Class of the Kirchhoff Wave Equations with Nonlinear Strongly Damped Terms
}

\author{
Chengfei Ai, Huixian Zhu, Guoguang Lin \\ Department of Mathematics, Yunnan University, Kunming, China \\ Email: aicf5206@163.com, zhuhx0917@163.com, gglin@ynu.edu.cn
}

How to cite this paper: Ai, C.F., Zhu, H.X. and Lin, G.G. (2016) Approximate Inertial Manifold for a Class of the Kirchhoff Wave Equations with Nonlinear Strongly Damped Terms. International Journal of Modern Nonlinear Theory and Application, 5, 218-234. http://dx.doi.org/10.4236/ijmnta.2016.54020

Received: October 8, 2016

Accepted: November 26, 2016

Published: November 29, 2016

Copyright $\odot 2016$ by authors and Scientific Research Publishing Inc. This work is licensed under the Creative Commons Attribution International License (CC BY 4.0).

http://creativecommons.org/licenses/by/4.0/ (c) (i) Open Access

\section{Abstract}

This paper is devoted to the long time behavior of the solution to the initial boundary value problems for a class of the Kirchhoff wave equations with nonlinear strongly damped terms: $u_{t t}-\varepsilon_{1} \Delta u_{t}+\alpha\left|u_{t}\right|^{p-1} u_{t}+\beta|u|^{q-1} u-\phi\left(\|\nabla u\|^{2}\right) \Delta u=f(x)$. Firstly, in order to prove the smoothing effect of the solution, we make efficient use of the analytic property of the semigroup generated by the principal operator of the equation in the phase space. Then we obtain the regularity of the global attractor and construct the approximate inertial manifold of the equation. Finally, we prove that arbitrary trajectory of the Kirchhoff wave equations goes into a small neighbourhood of the approximate inertial manifold after large time.

\section{Keywords}

Kirchhoff Wave Equation, Global Attractor, The Smoothing Effect, The Regularity, Approximate Inertial Manifold

\section{Introduction}

It is well known that we are studying the long time behavior of the infinite dimensional dynamical systems of the nonlinear partial differential equations, and the concept of the inertial manifold plays an important role in this field. In 1985, G. Foias, G. R. Sell and $\mathrm{R}$. Teman [1] first put forward the concept of the inertial manifold; it is an invariant finite dimensional Lipschitz manifold; it is exponentially attracting trajectory and contains the global attractor. But to ensure that existing conditions are very harsh for inertial manifolds (For instance, spectral interval condition), the existence of a large number of important partial differential equations is still not solved. Therefore, people na- 
turally think of using an approximate, smooth and easy to solve the manifolds to approximate the global attractor and inertial manifolds, which is the approximate inertial manifold.

Approximate inertial manifolds are finite dimensional smooth manifolds, and each solution of the equation is in a finite time to its narrow field. In particular, the global attractor is also included in its neighbourhood. The existence of approximate inertial manifolds of a large number of dissipative partial differential equations has been studied [2]-[7].

In this paper, we are concerned a class of the Kirchhoff wave equations with nonlinear strongly damped terms referred to as follows:

$$
\begin{gathered}
u_{t t}-\varepsilon_{1} \Delta u_{t}+\alpha\left|u_{t}\right|^{p-1} u_{t}+\beta|u|^{q-1} u-\phi\left(\|\nabla u\|^{2}\right) \Delta u=f(x) \quad \text { in } \Omega \times \mathbb{R}^{+}, \\
u(x, 0)=u_{0}(x) ; u_{t}(x, 0)=u_{1}(x), \quad x \in \Omega, \\
\left.u(x, t)\right|_{\partial \Omega}=0,\left.\Delta u(x, t)\right|_{\partial \Omega}=0, \quad x \in \Omega .
\end{gathered}
$$

where $\Omega$ is a bounded domain in $\mathbb{R}^{N}$ with smooth boundary $\partial \Omega$, and $\varepsilon_{1}, \alpha, \beta$ are positive constants, and the assumptions on $\phi\left(\|\nabla u\|^{2}\right)$ will be specified later.

In [8], G. Kirchhoff firstly proposed the so called Kirchhoff string model in the study nonlinear vibration of an elastic string. Kirchhoff type wave equations have been studied by many scholars (see [9] [10] [11]). In reference [12], the long time behavior of solutions for the initial value problems (1.1) - (1.3), the existence of global attractor corresponding to the semigroup operator $S(t)$ and the dimension estimation of global attractor, have been researched.

In [13], Dai Zhengde, Guo Boling, Lin Guoguang studied the fractal structure of attractor for the generalized Kuramoto-Sivashinsky equations:

$$
\begin{gathered}
u_{t}+\alpha u_{x x}+\beta u_{x x x}+\gamma u_{x x x x}+f(u)_{x}+\varphi(u)_{x x}=g(u)+h(x), t>0, x \in R, \\
u(x, 0)=u_{0}(x), \\
u(x-D, t)=u(x+D, t), t>0, x \in R .
\end{gathered}
$$

where $\alpha \geq 0, \gamma>0, D>0$.

In [14], Li Yongsheng, Zhang Weiguo studied regularity and approximate of the attractor for the strongly damped wave equation:

$$
\begin{gathered}
u_{t t}-\alpha u_{x x t}-\beta u_{x x}+h(u) u_{t}+f(u)=g(x), t>0, x \in(0,1), \\
u(0, t)=u(1, t)=0, t \geq 0, \\
u(x, 0)=u_{0}(x), u_{t}(x, 0)=u_{1}(x), x \in(0,1) .
\end{gathered}
$$

where $\alpha, \beta$ are positive constants.

Luo Hong, Pu Zhilin and Chen Guanggan [15] studied regularity of the attractor and approximate inertial manifold for strongly damped nonlinear wave equation:

$$
\begin{gathered}
u_{t t}-\alpha u_{x x t}-\sigma\left(u_{x}\right)_{x}+f(u)=g(x), x \in(0,1), t \in[0, \infty), \\
u(0)=u_{0}, u_{t}(0)=u_{1},
\end{gathered}
$$




$$
u(0, t)=u(1, t)=0 .
$$

where $\alpha$ is a positive constant.

Wang Lei, Dang Jinbao and Lin Guoguang [16] also studied the approximate inertial manifolds of the fractional nonlinear Schrödinger equation:

$$
\begin{gathered}
i u_{t}+(-\Delta)^{\alpha} u+\beta|u|^{\rho} u+i \delta u=f(x), x \in \Omega, t>0, \\
u(x, 0)=u_{0}(x), x \in \Omega, \\
u\left(x+L e_{i}, t\right)=u(x, t), x \in \Omega, t>0 .
\end{gathered}
$$

where $\Omega=(0, L)^{n}, e_{i}=(0, \cdots, 0,1,0, \cdots, 0),(i=1,2, \cdots, n)$ is a standard orthogonal base, $\mathrm{i}$ is the imaginary unit. $\alpha>\frac{n}{2}, \beta>0, \rho>0, \delta>0$.

Recently, Sufang Zhang, Jianwen Zhang [17] studied approximate inertial manifold of strongly damped wave equation:

$$
\begin{gathered}
u_{t t}-\Delta u-\Delta u_{t}-\alpha \Delta u_{t t}+f(u)=g(x, t) \in \Omega \times R^{+}, \\
u(x, 0)=u_{0}, u_{t}(x, 0)=u_{1}, x \in \Omega, \\
u(x, t)=0,(x, t) \in \partial \Omega \times R^{+} .
\end{gathered}
$$

where $\Omega$ is a bounded domain in $\mathbb{R}^{N}$ with smooth boundary $\partial \Omega, \alpha>0$ is a constant, the function $g \in L^{2}(\Omega)$.

There have many researches on approximate inertial manifolds for nonlinear wave equations (see [18]-[24]). In order to construct the approximate inertial manifolds for the initial boundary value problems, in the references [14] to [15], the regularity of the global attractor is studied, and then the approximate inertial manifold is constructed. In [18], Tian Lixin, Lin Yurui construct approximate inertial manifolds under spline wavelet basis in weakly damped forced $\mathrm{KdV}$ equation. In infinite-dimensional dynamical systems, Kirchhoff type wave equation is a class of very important equation. However, the approximate inertial manifold and inertial manifold of the Kirchhoff wave equation with nonlinear strong damping term are rarely studied. Based on the current research situation of Kirchhoff wave equations, in this paper, we first study the regularity of the global attractor for a class of the Kirchhoff wave equations with nonlinear strongly damped terms, and then construct its approximate inertial manifold.

The paper is arranged as follows. In Section 2, we state some assumptions, notations and the main results are stated. In Section 3, through the estimation of solution smoothness of higher order, then we obtain the regularity of the global attractor. In Section 4, by constructing a smooth manifold, namely the approximate inertial manifold, we approximate the global attractor for the problems (1.1) - (1.3).

\section{Statement of Some Assumptions, Notations and Main Results}

For convenience, we denote the norm and scalar product in $L^{2}(\Omega)$ by $\|$.$\| and (.,$.$) ;$ $f=f(x), \quad L^{p}=L^{p}(\Omega), \quad H^{k}=H^{k}(\Omega), \quad H_{0}^{k}=H_{0}^{k}(\Omega),\|\cdot\|=\|\cdot\|_{L^{2}},\|\cdot\|_{p}=\|\cdot\|_{L^{p}}$.

Let $E=L^{2}(\Omega)$, where $\Omega \subset \mathbb{R}^{N}$ is a bounded domain, where the norm is defined 
as $\|\cdot\| \cdot A=-\Delta$ is an unbounded positive definite self adjoint operator. Let $D(A)=H^{2}(\Omega) \cap H_{0}^{1}(\Omega)$, From reference [25], $A^{-1}$ is compact, $D(A)$ is dense in $E$, so $E=\operatorname{span}\left\{\omega_{k}\right\}_{k=1}^{\infty}$, where $E$ is space by $\left\{\omega_{1}, \omega_{2}, \cdots, \omega_{k} \cdots\right\}$ as base generated.

$A \omega_{k}=\lambda_{k} \omega_{k}$, where $\lambda_{k}, \omega_{k}$ are the eigenvalues and eigenvectors of $A$, $0<\lambda_{1} \leq \lambda_{2} \leq \lambda_{3} \leq \cdots, \lambda_{k} \rightarrow \infty, k=1,2,3, \cdots$. Then $\omega_{k}$ consists of a set of standard orthogonal basis space $E$.

We present some assumptions and notations needed in the proof of our results as follows:

$\left(\mathrm{G}_{1}\right)$ From reference [12], we set some constants: $\varepsilon_{1}>0, \varepsilon>0, \gamma_{1}>0, \gamma_{2}>0, K \geq 0$, such that $K-2 \varepsilon \geq 0, \quad \varepsilon_{1} \varepsilon \leq \phi\left(\|\nabla u\|^{2}\right) \leq \frac{\gamma_{1}}{K-2 \varepsilon}\left(1-\frac{K-2 \varepsilon}{\gamma_{1}} \mathrm{e}^{-(K-2 \varepsilon) t}\right)$.

$\left(\mathrm{G}_{2}\right)$ Let $\phi(s) \in C^{1}([0,+\infty))$, and $\phi(0)=0$, sup $\left|\phi^{\prime}(s)\right| \leq r_{0}, \quad \forall s \in[0,+\infty)$.

Theorem 2.1 From reference [12], due to $\left(\mathrm{G}_{1}\right),\left(\mathrm{G}_{2}\right)$ hold,

(i) Let $f(x) \in L^{2}(\Omega)$, then for each $u_{0} \in H^{2}(\Omega) \cap H_{0}^{1}(\Omega), u_{1} \in L^{2}(\Omega)$, the problems (1.1)-(1.3) exist solution $u, u \in C_{b}([0,+\infty) ; D(A))$; $u_{t} \in C_{b}([0,+\infty) ; E) \cap L^{2}\left(0, T ; H_{0}^{1}(\Omega)\right), \forall T>0$.

(ii) Let $f(x) \in H_{0}^{1}(\Omega), S(t)$ is the semigroup operator for the problems (1.1) (1.3), then the semigroup $S(t)$ exists a compact global attractor $\mathcal{A}_{0}$. So we can find a compact connected invariant set $\mathrm{B}$ to absorb all the bounded sets on $D(A) \times E$.

\section{The Regularity of Global Attractor}

In order to obtain the regularity of global attractor, we need to give a higher order uniform a priori estimates for the solution.

Let $v=u_{t}$, then the problem (1.1) can be reduced to the following form:

$$
\begin{gathered}
u_{t}=v, \\
v_{t}-\varepsilon_{1} \Delta v+\alpha|v|^{p-1} v+\beta|u|^{q-1} u-\phi\left(\|\nabla u\|^{2}\right) \Delta u=f(x) .
\end{gathered}
$$

Let

$$
U=\left(\begin{array}{l}
u \\
v
\end{array}\right), \Lambda=\left(\begin{array}{cc}
0 & -I \\
-\phi\left(\|\nabla u\|^{2}\right) \Delta & -\varepsilon_{1} \Delta
\end{array}\right), F U=\left(\begin{array}{c}
0 \\
F_{1}(u, v)
\end{array}\right), D(B)=[D(A)]^{2} .
$$

where $F_{1}(u, v)=f(x)-\alpha|v|^{p-1} v-\beta|u|^{q-1} u$.

Further, we rewrite the problems (1.1) - (1.3):

$$
\frac{\mathrm{d} U}{\mathrm{~d} t}+\Lambda U=F(U), U(0)=U_{0}=\left(\begin{array}{l}
u_{0} \\
u_{1}
\end{array}\right) \text {. }
$$

From references [26] [27], $\Lambda$ is a linear dense closed operator on $D(A) \times E$, which is a sector operator and has a bounded inverse. $\Lambda$ generates an analytic semigroup on $D(A) \times E$.

Lemma 3.1 From references [14] [15], due to $\left(\mathrm{G}_{1}\right),\left(\mathrm{G}_{2}\right)$ hold, let $f \in L^{2}(\Omega),\left.u(x, t)\right|_{\partial \Omega}=0$, then

Each $\left(u_{0}, u_{1}\right) \in D(A) \times E$, the solution to the problems (1.1) - (1.3) meet the follow- 
ing conditions:

$$
u, u_{t} \in C^{\theta}((0,+\infty) ; D(A)), u_{t t} \in C^{\theta}((0,+\infty) ; E), \forall \theta \in(0,1) .
$$

And there exist $\tau_{0}>0, K_{0}>0$ such that the following inequalities are established:

$$
\left\|u_{t}(t)\right\|_{D(A)} \leq K_{0},\left\|u_{t t}(t)\right\| \leq R_{1}, \forall t \geq K_{0} .
$$

where $D(A)=H^{2}(\Omega) \cap H_{0}^{1}(\Omega), \quad K_{0}$ is independent of the initial value $U_{0}$.

Proof. By the first conclusion (i) of theorem 2.1, when $u_{0} \in D(A), u_{1} \in E$, the solution $u$ meet: $u \in C_{b}([0,+\infty) ; D(A)), u_{t} \in C_{b}\left([0,+\infty) ; L^{2}(\Omega)\right)$,

$\forall T>0, u_{t} \in L^{2}\left(0, T ; H_{0}^{1}(\Omega)\right)$. By the second conclusion (ii) of theorem 2.1 , there exist $\tau>0, R_{0}>0$, when $t>\tau$,

$$
\|u\|_{D(A)} \leq R_{0},\left\|u_{t}\right\| \leq R_{0}
$$

Meanwhile, $\Delta u$ is uniformly bounded in $E, t \in[0,+\infty)$.

$$
F U=\left(0, F_{1}(u, v)\right)^{\mathrm{T}}=\left(0, f(x)-\alpha|v|^{p-1} v-\beta|u|^{q-1} u\right)^{\mathrm{T}} \in C_{b}([0,+\infty) ; D(A) \times E)
$$

Then $\quad F_{1}(u, v) \in C_{b}([0, T] ; D(A) \times E) \rightarrow L^{p}(0, T ; D(A) \times E), \quad p=\frac{1}{1-\theta}, \theta \in(0,1)$.

Based on the reference [27], the analytic properties of the semigroups generated by $\Lambda$ and the Equation (3.4), immediately get $\forall 0<t_{0}<T$, the solution

$U(\cdot) \in C^{\theta}\left(\left[t_{0}, T\right] ; D(A) \times E\right)$, furthermore, for the non-homogeneous term $F_{1}(u, v)$ in the Equation (3.4), $F_{1}(u, v) \in C^{\theta}\left(\left[t_{0}, T\right] ; D(A) \times E\right)$, then $U(\cdot) \in C^{\theta}\left(\left(t_{0}, T\right] ; D(\Lambda)\right)$, $U_{t}(\cdot), \Lambda U(\cdot) \in C^{\theta}\left(\left(t_{0}, T\right) ; D(A) \times E\right)$, due to $T, t_{0}$ are arbitrary, $U(\cdot) \in C^{\theta}((0,+\infty) ; D(\Lambda)), U_{t}(\cdot) \in C^{\theta}((0,+\infty) ; D(A) \times E)$.

Since $U(\tau) \in D(\Lambda), U_{t}(\tau) \in D(A) \times E$, we are now considering $\tau, U_{t}(\tau)$, respectively, as the initial time, initial value. Next, we consider the equation about $\mathcal{V}=U_{t}=\left(v, v_{t}\right)^{\mathrm{T}}$,

$$
\mathcal{V}_{t}+\Lambda \mathcal{V}=F(U)_{t}=\left(0,-\alpha\left(|v|^{p-1} v\right)_{t}-\beta\left(|u|^{q-1} u\right)_{t}\right)^{\mathrm{T}} .
$$

then

$$
\begin{gathered}
v_{t t}-\varepsilon_{1} \Delta v_{t}-\left(\phi\left(\|\nabla u\|^{2}\right) \Delta u\right)_{t}+\alpha\left(|v|^{p-1} v\right)_{t}+\beta\left(|u|^{q-1} u\right)_{t}=0, \\
v(x, \tau)=u_{t}(x, \tau) \in D(A), \\
v_{t}(x, \tau)=u_{t t}(x, \tau) \in E, \\
\left.v(x, t)\right|_{\partial \Omega}=0,\left.\Delta v(x, t)\right|_{\partial \Omega}=0, x \in \Omega, t \geq \tau .
\end{gathered}
$$

Next, we multiply $v_{t}+\varepsilon v$ with both sides of the equation (3.10) and integrate over $\Omega$ to obtain

$$
\begin{gathered}
\left(v_{t t}, v_{t}+\varepsilon v\right)=\frac{1}{2} \frac{\mathrm{d}}{\mathrm{d} t}\left\|v_{t}\right\|^{2}+\varepsilon \frac{\mathrm{d}}{\mathrm{d} t}\left(\int_{\Omega} v \cdot v_{t} \mathrm{~d} x\right)-\varepsilon\left\|v_{t}\right\|^{2} . \\
\left(-\varepsilon_{1} \Delta v_{t}, v_{t}+\varepsilon v\right)=\varepsilon_{1}\left\|\nabla v_{t}\right\|^{2}+\frac{\varepsilon_{1} \varepsilon}{2} \frac{\mathrm{d}}{\mathrm{d} t}\|\nabla v\|^{2} .
\end{gathered}
$$




$$
\begin{aligned}
& \left(-\left(\phi\left(\|\nabla u\|^{2}\right) \Delta u\right)_{t}, v_{t}+\varepsilon v\right) \\
& =\left(-\left(\phi\left(\|\nabla u\|^{2}\right) \Delta u\right)_{t}, v_{t}\right)+\left(-\left(\phi\left(\|\nabla u\|^{2}\right) \Delta u\right)_{t}, \varepsilon v\right)
\end{aligned}
$$

where from the hypothesis (G2),

$$
\begin{gathered}
\left(-\left(\phi\left(\|\nabla u\|^{2}\right) \Delta u\right)_{t}, v_{t}\right) \\
=-\phi\left(\|\nabla u\|^{2}\right)_{t} \int_{\Omega} \Delta u v_{t} \mathrm{~d} x+\frac{\mathrm{d}}{\mathrm{d} t}\left[\frac{1}{2} \phi\left(\|\nabla u\|^{2}\right)\|\nabla v\|^{2}\right]-\frac{1}{2} \phi\left(\|\nabla u\|^{2}\right)_{t} \cdot\|\nabla v\|^{2} \\
\geq-\frac{r_{0}}{2}\|\nabla u\|^{2}-\frac{r_{0}}{2}\left\|\nabla v_{t}\right\|^{2}+\frac{\mathrm{d}}{\mathrm{d} t}\left[\frac{1}{2} \phi\left(\|\nabla u\|^{2}\right)\|\nabla v\|^{2}\right]-\frac{r_{0}}{2}\|\nabla v\|^{2} . \\
\left(-\left(\phi\left(\|\nabla u\|^{2}\right) \Delta u\right)_{t}, \varepsilon v\right) \\
=-\varepsilon \phi\left(\|\nabla u\|^{2}\right) \cdot(\Delta u, v)+\varepsilon \phi\left(\|\nabla u\|^{2}\right)\|\nabla v\|^{2} \\
\geq-\frac{r_{0} \varepsilon}{2}\|\nabla u\|^{2}-\frac{r_{0} \varepsilon}{2}\|\nabla v\|^{2}+\varepsilon_{1} \varepsilon^{2}\|\nabla v\|^{2} . \\
\alpha\left(\left(|v|^{p-1} v\right)_{t}, v_{t}+\varepsilon v\right)=\alpha\left(\left(|v|^{p-1} v\right)_{t}, v_{t}\right)+\alpha \varepsilon\left(\left(|v|^{p-1} v\right)_{t}, v\right) \\
\alpha\left(\left(|v|^{p-1} v\right)_{t}, v_{t}\right)=\int_{\Omega}|v|^{p-1} v_{t} v_{t} \mathrm{~d} x+\int_{\Omega}\left(\left(v^{2}\right)^{\frac{p-1}{2}}\right)_{t} v v_{t} \mathrm{~d} x \\
=\alpha \int_{\Omega}|v|^{p-1} v_{t} v_{t} \mathrm{~d} x+\alpha \int_{\Omega} \frac{p-1}{2}\left(\left(v^{2}\right)^{\frac{p-3}{2}}\right) 2 v v_{t} v v_{t} \mathrm{~d} x \\
=\alpha \int_{\Omega}|v|^{p-1} v_{t} v_{t} \mathrm{~d} x+\alpha(p-1) \int_{\Omega}|v|^{p-1} v_{t} v_{t} \mathrm{~d} x \\
=\alpha p \int_{\Omega}|v|^{p-1} v_{t}^{2} \mathrm{~d} x . \\
\alpha \varepsilon\left(\left(|v|^{p-1} v\right)_{t}, v\right)=\alpha \varepsilon \frac{\mathrm{d}}{\mathrm{d} t}\left(\int_{\Omega}|v|^{p-1} v v \mathrm{~d} x\right)-\alpha \varepsilon \int_{\Omega}|v|^{p-1} v v_{t} \mathrm{~d} x .
\end{gathered}
$$

where $\alpha \varepsilon \int_{\Omega}|v|^{p-1} v v_{t} \mathrm{~d} x \leq \alpha \varepsilon\left(\int_{\Omega}|v|^{2 p} \mathrm{~d} x\right)^{\frac{1}{2}} \cdot\left(\int_{\Omega} v_{t}^{2} \mathrm{~d} x\right)^{\frac{1}{2}}=\alpha \varepsilon\|v\|_{2 p}^{p}\left\|v_{t}\right\|$.

By using Gagliardo-Nirenberg's embedding inequality, Hölder's inequality:

$$
\begin{aligned}
\|v\|_{2 p}^{p}\left\|v_{t}\right\| & \leq C_{1}\|\nabla v\|^{\frac{(p-1) n}{2}}\|v\|^{2 p-(p-1) n}\left\|v_{t}\right\| \\
& \leq \frac{C_{1}^{2} \alpha \varepsilon\left\|v_{t}\right\|^{2}}{2}+\frac{\|\nabla v\|^{(p-1) n}\|v\|^{2 p-(p-1) n}}{2 \alpha \varepsilon} \\
& \leq \frac{C_{1}^{2} \alpha \varepsilon\left\|v_{t}\right\|^{2}}{2}+\frac{C_{2}\|v\|^{2}}{4 \alpha \varepsilon}+\frac{C_{2}\|\nabla v\|^{2}}{4 \alpha \varepsilon}+C_{3}\left(C_{2}, \alpha, \varepsilon\right)
\end{aligned}
$$

Similar to the relation (3.20):

$$
\beta\left(\left(|u|^{q-1} u\right)_{t}, v_{t}+\varepsilon v\right)=\beta q \int_{\Omega}|u|^{q-1} v v_{t} \mathrm{~d} x+\beta q \varepsilon \int_{\Omega}|u|^{q-1} v^{2} \mathrm{~d} x .
$$

By using Hölder's inequality, Young's inequality and Sobolev's embedding inequality: 


$$
\begin{gathered}
\beta q \int_{\Omega}|u|^{q-1} v v_{t} \mathrm{~d} x \leq \beta q \int_{\Omega}|u|^{q-1}|v|\left|v_{t}\right| \mathrm{d} x \leq \beta q \int_{\Omega}|u|^{q-1}\left(\frac{|v|^{2}}{2}+\frac{\left|v_{t}\right|^{2}}{2}\right) \mathrm{d} x . \\
\frac{\beta q}{2} \int_{\Omega}|u|^{q-1}\left|v_{t}\right|^{2} \mathrm{~d} x \leq \frac{\beta q}{2}\left(\int_{\Omega}|u|^{2(q-1)} \mathrm{d} x\right)^{\frac{1}{2}}\left(\int_{\Omega}\left|v_{t}\right|^{4} \mathrm{~d} x\right)^{\frac{1}{2}}=\frac{\beta q}{2}\|u\|_{2(q-1)}^{q-1}\left\|v_{t}\right\|_{4}^{2} . \\
\|u\|_{2(q-1)}^{q-1} \leq C_{4}\|\Delta u\|^{\frac{n(q-2)}{4}}\|u\|^{\frac{4(q-1)-n(q-2)}{4}} ; \\
\left\|v_{t}\right\|_{4}^{2} \leq C_{5}\left\|\nabla v_{t}\right\|^{\frac{n}{2}}\left\|v_{t}\right\|^{\frac{4-n}{2}} \leq \frac{\varepsilon_{2}\left\|\nabla v_{t}\right\|^{2}}{2}+\frac{\left\|v_{t}\right\|^{2}}{2 \varepsilon_{2}}+C_{6}\left(\varepsilon_{2}, C_{5}\right) .
\end{gathered}
$$

In reference [12], $\|\Delta u\|,\|u\|$ are bounded by a priori estimates.

$$
\|u\|_{2(q-1)}^{q-1}\left\|v_{t}\right\|_{4}^{2} \leq C_{7}\left(C_{4},\|u\|_{\infty},\|\Delta u\|_{\infty}\right)\left(\frac{\varepsilon_{2}\left\|\nabla v_{t}\right\|^{2}}{2}+\frac{\left\|v_{t}\right\|^{2}}{2 \varepsilon_{2}}+C_{6}\right) .
$$

So we get:

$$
\begin{aligned}
\beta\left(\left(|u|^{q-1} u\right)_{t}, v_{t}+\varepsilon v\right) \geq & \left(\beta q \varepsilon-\frac{\beta q}{2}\right) \int_{\Omega}|u|^{q-1} v^{2} \mathrm{~d} x-\frac{\beta q C_{7} \varepsilon_{2}}{2}\left\|\nabla v_{t}\right\|^{2} \\
& -\frac{\beta q C_{7}}{2 \varepsilon_{2}}\left\|v_{t}\right\|^{2}-\beta q C_{7} C_{6} .
\end{aligned}
$$

From above, we have

$$
\begin{gathered}
\Phi_{1}=\frac{1}{2}\left\|v_{t}\right\|^{2}+\varepsilon \int_{\Omega} v v_{t} \mathrm{~d} x+\frac{\varepsilon \varepsilon_{1}}{2}\|\nabla v\|^{2}+\alpha \varepsilon \int_{\Omega}|v|^{p-1} v^{2} \mathrm{~d} x+\frac{1}{2} \phi\left(\|\nabla u\|^{2}\right)\|\nabla v\|^{2} . \\
\Psi_{1}=\varepsilon_{1}\left\|\nabla v_{t}\right\|^{2}-\varepsilon\left\|v_{t}\right\|^{2}-\phi\left(\|\nabla u\|^{2}\right)_{t} \int_{\Omega} \Delta u v_{t} \mathrm{~d} x-\frac{1}{2} \phi\left(\|\nabla u\|^{2}\right)_{t} \cdot\|\nabla v\|^{2} \\
-\varepsilon \phi\left(\|\nabla u\|^{2}\right)_{t}(\Delta u, v)+\varepsilon \phi\left(\|\nabla u\|^{2}\right)\|\nabla v\|^{2}+\alpha p \int_{\Omega}|v|^{p-1} v_{t}^{2} \mathrm{~d} x \\
+\beta q \varepsilon \int_{\Omega}|u|^{q-1} v^{2} \mathrm{~d} x-\alpha \varepsilon \int_{\Omega}|v|^{p-1} v v_{t} \mathrm{~d} x+\beta q \int_{\Omega}|u|^{q-1} v v_{t} \mathrm{~d} x .
\end{gathered}
$$

Taking $\kappa_{1}>0$, then

$$
\begin{gathered}
\Psi_{1}-\kappa_{1} \Phi_{1} \geq \varepsilon_{1}\left\|\nabla v_{t}\right\|^{2}-\varepsilon\left\|v_{t}\right\|^{2}-\frac{r_{0}}{2}\|\Delta u\|^{2}-\frac{r_{0}}{2}\left\|v_{t}\right\|^{2}-\frac{r_{0}}{2}\|\nabla v\|^{2}-\frac{r_{0} \varepsilon}{2}\|\nabla u\|^{2} \\
-\frac{r_{0} \varepsilon}{2}\|\nabla v\|^{2}+\varepsilon^{2} \varepsilon_{1}\|\nabla v\|^{2}-\frac{C_{1}^{2} \alpha^{2} \varepsilon^{2}}{2}\left\|v_{t}\right\|^{2}-\frac{C_{2}\|v\|^{2}}{4}-\frac{C_{2}\|\nabla v\|^{2}}{4} \\
-C_{3}-\frac{\beta q C_{7} \varepsilon_{2}}{2}\left\|\nabla v_{t}\right\|^{2}-\frac{\beta q C_{7}}{2 \varepsilon_{2}}\left\|v_{t}\right\|^{2}-\beta q C_{7} C_{6}-\frac{\kappa_{1}\left\|v_{t}\right\|^{2}}{2} \\
-\frac{\kappa_{1} \varepsilon\|v\|^{2}}{2}-\kappa_{1} \alpha \varepsilon \int_{\Omega}|v|^{p-1} v^{2} \mathrm{~d} x-\frac{\kappa_{1} \varepsilon_{1} \varepsilon\|\nabla v\|^{2}}{2}-\frac{\kappa_{1} \gamma_{1}\|\nabla v\|^{2}}{2(K-2 \varepsilon)} . \\
\int_{\Omega}|v|^{p-1} v^{2} \mathrm{~d} x \leq(|\Omega|)^{\frac{1}{2}}\|v\|_{2(p+1)}^{p+1} \leq C_{8}\left((|\Omega|)^{\frac{1}{2}}\right)\|\nabla v\|^{\frac{n p}{2}}\|v\|^{\frac{2 p+2-n p}{2}} \\
\leq C_{9} \frac{\|v\|^{2}}{2}+C_{9} \frac{\|\nabla v\|^{2}}{2}+C_{10}\left(C_{8}, C_{9}\right) .
\end{gathered}
$$

At last, we get: 


$$
\begin{aligned}
\Psi_{1}-\kappa_{1} \Phi_{1} \geq & \left(\varepsilon_{1}-\frac{C_{7} \beta q \varepsilon_{2}}{2}\right)\left\|\nabla v_{t}\right\|^{2}-\left(\varepsilon+\frac{r_{0}}{2}+\frac{C_{1}^{2} \alpha^{2} \varepsilon^{2}}{2}+\frac{C_{7} \beta q}{2 \varepsilon_{2}}+\frac{\kappa_{1}}{2}+\frac{\kappa_{1} \varepsilon}{2}\right)\left\|v_{t}\right\|^{2} \\
& +\left(\varepsilon^{2} \varepsilon_{1}-\frac{r_{0}}{2}-\frac{r_{0} \varepsilon}{2}-\frac{\kappa_{1} \varepsilon_{1} \varepsilon}{2}-\frac{C_{9} \kappa_{1} \alpha \varepsilon}{2}-\frac{C_{2}}{4}-\frac{\kappa_{1} \gamma_{1}}{2(K-2 \varepsilon)}\right)\|\nabla v\|^{2} \\
& -\left(\frac{C_{2}}{4}+\frac{\kappa_{1} \varepsilon}{2}+\frac{C_{9} \kappa_{1} \alpha \varepsilon}{2}\right)\|v\|^{2}-C .
\end{aligned}
$$

Let $m_{1}=\varepsilon_{1}-\frac{C_{7} \beta q \varepsilon_{2}}{2} ; m_{2}=\varepsilon+\frac{r_{0}}{2}+\frac{C_{1}^{2} \alpha^{2} \varepsilon^{2}}{2}+\frac{C_{7} \beta q}{2 \varepsilon_{2}}+\frac{\kappa_{1}}{2}+\frac{\kappa_{1} \varepsilon}{2}$;

$$
\begin{gathered}
m_{3}=\varepsilon^{2} \varepsilon_{1}-\frac{r_{0}}{2}-\frac{r_{0} \varepsilon}{2}-\frac{\kappa_{1} \varepsilon_{1} \varepsilon}{2}-\frac{C_{9} \kappa_{1} \alpha \varepsilon}{2}-\frac{C_{2}}{4}-\frac{\kappa_{1} \gamma_{1}}{2(K-2 \varepsilon)} ; \\
m_{4}=\frac{C_{2}}{4}+\frac{\kappa_{1} \varepsilon}{2}+\frac{C_{9} \kappa_{1} \alpha \varepsilon}{2} .
\end{gathered}
$$

By using Poincaré's inequality, we get

$$
\Psi_{1}-\kappa_{1} \Phi_{1} \geq\left(\lambda_{1} m_{1}-m_{2}\right)\left\|\nabla v_{t}\right\|^{2}+\left(\lambda_{1} m_{3}-m_{4}\right)\|v\|^{2}-C .
$$

We take proper $\varepsilon, \varepsilon_{1}, \varepsilon_{2}, \gamma_{1}, \kappa_{1}, r_{0}, \alpha, \beta$, such that:

$$
\left\{\begin{array}{l}
\lambda_{1} m_{1}-m_{2} \geq 0 \\
\lambda_{1} m_{3}-m_{4} \geq 0 .
\end{array}\right.
$$

Then

$$
\Psi_{1}-\kappa_{1} \Phi_{1} \geq-C .
$$

From the relation (3.36), we can get

$$
\frac{\mathrm{d}}{\mathrm{d} t} \Phi_{1}(t)+\kappa_{1} \Phi_{1}(t) \leq C, t \geq \tau .
$$

By using Gronwall's inequality, we obtain:

$$
\Phi_{1}(t) \leq \Phi_{1}(\tau) \mathrm{e}^{-\kappa_{1}(t-\tau)}+\frac{C}{\kappa_{1}}\left(1-\mathrm{e}^{-\kappa_{1}(t-\tau)}\right), t \geq \tau .
$$

Taking $\tau_{0} \gg \tau$, such that $\Phi_{1}(\tau) \mathrm{e}^{-\kappa_{1}(t-\tau)} \leq 1$, then

$$
\Phi_{1}(t) \leq 1+\frac{C}{\kappa_{1}}, \forall t \geq \tau_{0}
$$

where

$$
\begin{aligned}
\Phi_{1} & =\frac{1}{2}\left\|v_{t}\right\|^{2}+\varepsilon \int_{\Omega} v v_{t} \mathrm{~d} x+\frac{\varepsilon \varepsilon_{1}}{2}\|\nabla v\|^{2}+\alpha \varepsilon \int_{\Omega}|v|^{p-1} v^{2} \mathrm{~d} x+\frac{1}{2} \phi\left(\|\nabla u\|^{2}\right)\|\nabla v\|^{2} \\
& \geq \frac{1}{2}\left\|v_{t}\right\|^{2}+\varepsilon \int_{\Omega} v v_{t} \mathrm{~d} x+\frac{\varepsilon \varepsilon_{1}}{2}\|\nabla v\|^{2}+\alpha \varepsilon \int_{\Omega}|v|^{p-1} v^{2} \mathrm{~d} x+\frac{1}{2} \phi\left(\|\nabla u\|^{2}\right)\|\nabla v\|^{2} \\
& \geq \frac{(1-\varepsilon)}{2}\left\|v_{t}\right\|^{2}+\frac{\left(2 \mu_{1} \varepsilon_{1} \varepsilon-C_{9} \mu_{1} \alpha \varepsilon-\varepsilon-C_{9} \alpha \varepsilon\right)}{2}\|v\|^{2}-\frac{C}{\kappa_{1}}
\end{aligned}
$$

Meanwhile, we once again take proper $\varepsilon, \varepsilon_{1}, \mu_{1}, \alpha$, such that: 


$$
\left\{\begin{array}{l}
1-\varepsilon>0 \\
2 \mu_{1} \varepsilon_{1} \varepsilon-C_{9} \mu_{1} \alpha \varepsilon-\varepsilon-C_{9} \alpha \varepsilon>0 .
\end{array}\right.
$$

So there are $\tau_{0}>0, K_{0}>0$, which make the following inequalities:

$$
\left\|u_{t}(t)\right\|_{D(A)} \leq K_{0},\left\|u_{t t}(t)\right\| \leq K_{0}, \forall t \geq \tau_{0} .
$$

where $D(A)=H^{2}(\Omega) \cap H_{0}^{1}(\Omega), \quad K_{0}$ is independent of the initial value $U_{0}$.

Lemma 3.2 From references [14] [15], due to $\left(\mathrm{G}_{1}\right),\left(\mathrm{G}_{2}\right)$ hold, let $f(0)=0$, $f \in D(A)=H^{2}(\Omega) \cap H_{0}^{1}(\Omega)$, then $\forall\left(u_{0}, u_{1}\right) \in D(A) \times E$, the solution to the problems (1.1) (1.3) meet the following conditions:

$$
u, u_{t} \in C^{\theta}\left((0,+\infty) ; D\left(A^{2}\right)\right), u_{t t} \in C^{\theta}((0,+\infty) ; D(A)), \forall \theta \in(0,1) .
$$

And there exist $\tau_{1}>0, K_{1}>0$ such that the following inequalities are established:

$$
\|u(t)\|_{D\left(A^{2}\right)} \leq K_{1},\left\|u_{t}(t)\right\|_{D\left(A^{2}\right)} \leq K_{1}, \forall t \geq \tau_{1} \text {. }
$$

Proof. Take proper $T$, such that $\forall 0<t_{0}<T, U\left(t_{0}\right) \in D(A)$, we are now considering the Equation (3.9), assume $\left(\mathrm{G}_{1}\right),\left(\mathrm{G}_{2}\right)$ hold, $f \in D(A), u, u_{t} \in C^{\theta}\left(\left[t_{0}, T\right] ; D(A)\right)$, $u_{t t} \in C^{\theta}\left(\left[t_{0}, T\right] ; E\right)$, the nonlinear term $F(U(t))_{t} \in C^{\theta}\left(\left[t_{0}, T\right] ; D(A) \times E\right)$. Based on the reference [27], the solution to the Equation (3.9):

$\mathcal{V}(\cdot), \mathcal{V}_{t}(\cdot), \Lambda \mathcal{V}(\cdot) \in C^{\theta}\left(\left[t_{0}, T\right] ; D(A) \times E\right)$. From (3.4), we get $U(\cdot) \in C^{\theta}\left(\left[t_{0}, T\right] ; D\left(\Lambda^{2}\right)\right)$, due to $T, t_{0}$ are arbitrary, $U(\cdot) \in C^{\theta}\left((0,+\infty) ; D\left(\Lambda^{2}\right)\right)$, $U(\cdot)_{t} \in C^{\theta}((0,+\infty) ; D(\Lambda))$, and then we can get $u, u_{t} \in C^{\theta}\left((0,+\infty) ; D\left(A^{2}\right)\right)$, $u_{t t} \in C^{\theta}((0,+\infty) ; D(A)), \quad \forall \theta \in(0,1)$.

Similar to lemma (3.1), we are now considering $\tau_{0}, U_{t}\left(\tau_{0}\right)$, respectively, as the initial time, initial value. Next, and once again, we consider the Equations (3.9) - (3.13), multiplying $-\Delta v_{t}-\varepsilon \Delta v$ with both sides of the equation (3.10) and integrating over $\Omega$ to obtain

$$
\begin{gathered}
\left(v_{t t},-\Delta v_{t}-\varepsilon \Delta v\right)=\frac{1}{2} \frac{\mathrm{d}}{\mathrm{d} t}\left\|\nabla v_{t}\right\|^{2}+\varepsilon \frac{\mathrm{d}}{\mathrm{d} t}\left(\int_{\Omega} \nabla v \cdot \nabla v_{t} \mathrm{~d} x\right)-\varepsilon\left\|\nabla v_{t}\right\|^{2} . \\
\left(-\varepsilon_{1} \Delta v_{t},-\Delta v_{t}-\varepsilon \Delta v\right)=\varepsilon_{1}\left\|\Delta v_{t}\right\|^{2}+\frac{\varepsilon_{1} \varepsilon}{2} \frac{\mathrm{d}}{\mathrm{d} t}\|\Delta v\|^{2} . \\
\left(-\left(\phi\left(\|\nabla u\|^{2}\right) \Delta u\right)_{t},-\Delta v_{t}-\varepsilon \Delta v\right) \\
=\left(-\left(\phi\left(\|\nabla u\|^{2}\right) \Delta u\right)_{t},-\Delta v_{t}\right)+\left(-\left(\phi\left(\|\nabla u\|^{2}\right) \Delta u\right)_{t},-\varepsilon \Delta v\right)
\end{gathered}
$$

where from the hypothesis (G2),

$$
\begin{aligned}
& \left(-\left(\phi\left(\|\nabla u\|^{2}\right) \Delta u\right)_{t},-\Delta v_{t}\right) \\
& =\left(\phi\left(\|\nabla u\|^{2}\right)\right)_{t}\left(\Delta u, \Delta v_{t}\right)+\frac{1}{2} \frac{\mathrm{d}}{\mathrm{d} t}\left[\phi\left(\|\nabla u\|^{2}\right)\|\Delta v\|^{2}\right]-\frac{1}{2}\left(\phi\left(\|\nabla u\|^{2}\right)\right)_{t}\|\Delta v\|^{2} \\
& \geq-\frac{r_{0}}{2}\|\Delta u\|^{2}-\frac{r_{0}}{2}\left\|\Delta v_{t}\right\|^{2}+\frac{1}{2} \frac{\mathrm{d}}{\mathrm{d} t}\left[\phi\left(\|\nabla u\|^{2}\right)\|\Delta v\|^{2}\right]-\frac{r_{0}}{2}\|\Delta v\|^{2} .
\end{aligned}
$$




$$
\begin{aligned}
& \left(-\left(\phi\left(\|\nabla u\|^{2}\right) \Delta u\right)_{t},-\varepsilon \Delta v\right) \\
& =\left(\phi\left(\|\nabla u\|^{2}\right)\right)_{t}(\Delta u, \varepsilon \Delta v)+\varepsilon \phi\left(\|\nabla u\|^{2}\right)\|\Delta v\|^{2} \\
& \geq-\frac{r_{0} \varepsilon}{2}\|\Delta u\|^{2}-\frac{r_{0} \varepsilon}{2}\|\Delta v\|^{2}+\varepsilon^{2} \varepsilon_{1}\|\Delta v\|^{2} .
\end{aligned}
$$

Similar to lemma 3.1

$$
\begin{aligned}
& \alpha\left(\left(|v|^{p-1} v\right)_{t},-\Delta v_{t}-\varepsilon \Delta v\right) \\
& =\alpha\left(\left(|v|^{p-1} v\right)_{t},-\Delta v_{t}\right)+\alpha\left(\left(|v|^{p-1} v\right)_{t},-\varepsilon \Delta v\right) \\
& =-\alpha p \int_{\Omega}|v|^{p-1} v_{t} \Delta v_{t} \mathrm{~d} x-\alpha p \varepsilon \int_{\Omega}|v|^{p-1} v_{t} \Delta v \mathrm{~d} x \\
& \beta\left(\left(|u|^{q-1} u\right)_{t},-\Delta v_{t}-\varepsilon \Delta v\right) \\
& =\beta\left(\left(|u|^{q-1} u\right)_{t},-\Delta v_{t}\right)+\beta\left(\left(|u|^{q-1} u\right)_{t},-\varepsilon \Delta v\right) \\
& =-\beta q \int_{\Omega}|u|^{q-1} v \Delta v_{t} \mathrm{~d} x-\beta q \varepsilon \int_{\Omega}|u|^{q-1} v \Delta v \mathrm{~d} x .
\end{aligned}
$$

By using Hölder's inequality, Young's inequality and Sobolev's embedding inequality:

$$
\begin{aligned}
& \alpha p \int_{\Omega}|v|^{p-1} v_{t} \Delta v_{t} \mathrm{~d} x \\
& \leq \alpha p\left(\int_{\Omega}|v|^{2(p-1)}\left|\Delta v_{t}\right| \mathrm{d} x\right)^{\frac{1}{2}}\left(\int_{\Omega}\left|\Delta v_{t}\right|\left|v_{t}\right|^{2} \mathrm{~d} x\right)^{\frac{1}{2}} \\
& \leq \frac{\alpha p}{2} \int_{\Omega}|v|^{2(p-1)}\left|\Delta v_{t}\right| \mathrm{d} x+\frac{\alpha p}{2} \int_{\Omega}\left|\Delta v_{t}\right|\left|v_{t}\right|^{2} \mathrm{~d} x \\
& \leq \frac{\alpha p}{2}\left(\int_{\Omega}|v|^{4(p-1)} \mathrm{d} x\right)^{\frac{1}{2}}\left(\int_{\Omega}\left|\Delta v_{t}\right|^{2} \mathrm{~d} x\right)^{\frac{1}{2}}+\frac{\alpha p}{2}\left(\int_{\Omega}\left|v_{t}\right|^{4} \mathrm{~d} x\right)^{\frac{1}{2}}\left(\int_{\Omega}\left|\Delta v_{t}\right|^{2} \mathrm{~d} x\right)^{\frac{1}{2}} \\
& =\frac{\alpha p}{2}\|v\|_{4(p-1)}^{2(p-1)}\left\|\Delta v_{t}\right\|+\frac{\alpha p}{2}\left\|v_{t}\right\|_{4}^{2}\left\|\Delta v_{t}\right\| \text {. } \\
& \frac{\alpha p}{2}\|v\|_{4(p-1))}^{2(p-1)}\left\|\Delta v_{t}\right\| \\
& \leq \frac{\alpha p}{2} C_{1}\left(\|v\|_{\infty}\right)\|\Delta v\|^{\frac{(2 p-3) n}{4}}\left\|\Delta v_{t}\right\| \\
& \leq \frac{r_{0}\|\Delta v\|^{2}}{8}+C_{2}\left(C_{1}, \alpha, p, r_{0}\right)+\frac{\alpha p\left\|\Delta v_{t}\right\|^{2}}{4} \\
& \frac{\alpha p}{2}\left\|v_{t}\right\|_{4}^{2}\left\|\Delta v_{t}\right\| \\
& \leq \frac{\alpha p}{4} C_{3}\left(\left\|v_{t}\right\|_{\infty}\right)\left\|\nabla v_{t}\right\|^{n}+\frac{\alpha p}{4}\left\|\Delta v_{t}\right\|^{2} \\
& \leq \frac{\varepsilon_{1}\left\|\nabla v_{t}\right\|^{2}}{8}+C_{4}\left(C_{3}, \alpha, \varepsilon_{1}, p\right)+\frac{\alpha p}{4}\left\|\Delta v_{t}\right\|^{2} . \\
& \alpha p \int_{\Omega}|v|^{p-1} v_{t} \Delta v_{t} \mathrm{~d} x \\
& \leq \frac{r_{0}\|\Delta v\|^{2}}{8}+C_{2}\left(C_{1}, \alpha, p, r_{0}\right)+\frac{\alpha p\left\|\Delta v_{t}\right\|^{2}}{2}+\frac{\varepsilon_{1}\left\|\nabla v_{t}\right\|^{2}}{8}+C_{4}\left(C_{3}, \alpha, \varepsilon_{1}, p\right)
\end{aligned}
$$


Through similar methods above

$$
\begin{aligned}
& \alpha p \varepsilon \int_{\Omega}|v|^{p-1} v_{t} \Delta v \mathrm{~d} x \\
\leq & \frac{\alpha p \varepsilon}{2} C_{5}\left(\|v\|_{\infty}\right)\|\Delta v\|^{\frac{(2 p-3) n}{4}}\|\Delta v\|+\frac{\alpha p \varepsilon}{4} C_{6}\left(\left\|v_{t}\right\|_{\infty}\right)\left\|\nabla v_{t}\right\|^{n}+\frac{\alpha p \varepsilon}{4}\|\Delta v\|^{2} \\
\leq & \frac{r_{0}\|\Delta v\|^{2}}{4}+C_{7}\left(C_{5}, \alpha, p, \varepsilon, r_{0}\right)+\frac{\alpha p \varepsilon}{4}\|\Delta v\|^{2}+\frac{\varepsilon_{1}\left\|\nabla v_{t}\right\|^{2}}{8}+C_{8}\left(C_{6}, \alpha, \varepsilon, \varepsilon_{1}, p\right) \\
& \beta q \int_{\Omega}|u|^{q-1} v \Delta v_{t} \mathrm{~d} x \\
& \leq \frac{\beta q}{4} C_{9}\left(\|u\|_{\infty}\right)\|\Delta u\|^{\frac{(2 q-3) n}{2}}+\frac{\beta q}{4}\left\|\Delta v_{t}\right\|^{2}+\frac{\beta q}{4} C_{10}\left(\|v\|_{\infty}\right)\|\nabla v\|^{n}+\frac{\beta q}{4}\left\|\Delta v_{t}\right\|^{2} \\
& \leq \frac{\beta q}{8}\|\Delta u\|^{2}+C_{11}\left(C_{9}, \beta, q\right)+\frac{\beta q}{2}\left\|\Delta v_{t}\right\|^{2}+\frac{r_{0}\|\nabla v\|^{2}}{8}+C_{12}\left(C_{10}, \beta, q, r_{0}\right) . \\
\beta q \varepsilon \int_{\Omega}|u|^{q-1} v \Delta v \mathrm{~d} x & \\
\leq & \frac{\beta q \varepsilon}{4} C_{13}\left(\|u\|_{\infty}\right)\|\Delta u\|^{\frac{(2 q-3) n}{2}}+\frac{\beta q \varepsilon}{4}\|\Delta v\|^{2}+\frac{\beta q \varepsilon}{4} C_{14}\left(\|v\|_{\infty}\right)\|\nabla v\|^{n}+\frac{\beta q \varepsilon}{4}\|\Delta v\|^{2} \\
\leq & \frac{\beta q \varepsilon}{8}\|\Delta u\|^{2}+C_{15}\left(C_{13}, \beta, q, \varepsilon\right)+\frac{\beta q \varepsilon}{2}\|\Delta v\|^{2}+\frac{r_{0}\|\nabla v\|^{2}}{8}+C_{16}\left(C_{14}, \beta, q, \varepsilon, r_{0}\right) .
\end{aligned}
$$

From above, we have

$$
\begin{aligned}
\Phi_{2} & =\frac{1}{2}\left\|\nabla v_{t}\right\|^{2}+\varepsilon \int_{\Omega} \nabla v \nabla v_{t} \mathrm{~d} x+\frac{\varepsilon \varepsilon_{1}}{2}\|\Delta v\|^{2}+\frac{1}{2} \phi\left(\|\nabla u\|^{2}\right)\|\Delta v\|^{2} . \\
\Psi_{2}= & \varepsilon_{1}\left\|\Delta v_{t}\right\|^{2}-\varepsilon\left\|\nabla v_{t}\right\|^{2}+\phi\left(\|\nabla u\|^{2}\right)_{t} \int_{\Omega} \Delta u \Delta v_{t} \mathrm{~d} x-\frac{1}{2} \phi\left(\|\nabla u\|^{2}\right) \cdot\|\Delta v\|^{2} \\
& +\varepsilon \phi\left(\|\nabla u\|^{2}\right)_{t}(\Delta u, \Delta v)+\varepsilon \phi\left(\|\nabla u\|^{2}\right)\|\Delta v\|^{2}-\beta q \varepsilon \int_{\Omega}|u|^{q-1} v \Delta v \mathrm{~d} x \\
& -\beta q \int_{\Omega}|u|^{q-1} v \Delta v_{t} \mathrm{~d} x-\alpha p \int_{\Omega}|v|^{p-1} v_{t} \Delta v_{t} \mathrm{~d} x-\alpha p \varepsilon \int_{\Omega}|v|^{p-1} v_{t} \Delta v \mathrm{~d} x .
\end{aligned}
$$

Taking $\kappa_{2}>0$, then

$$
\kappa_{2} \Phi_{2} \leq \frac{\kappa_{2}}{2}\left\|\nabla v_{t}\right\|^{2}+\frac{\kappa_{2} \varepsilon}{2}\|\nabla v\|^{2}+\frac{\kappa_{2} \varepsilon}{2}\left\|\nabla v_{t}\right\|^{2}+\frac{\kappa_{2} \gamma_{1}}{2(K-2 \varepsilon)}\|\Delta v\|^{2} .
$$

At last, we get:

$$
\begin{aligned}
\Psi_{2}-\kappa_{2} \Phi_{2} \geq & \left(\varepsilon_{1}-\frac{r_{0}}{2}-\frac{\beta q}{2}-\frac{\alpha p}{2}\right)\left\|\Delta v_{t}\right\|^{2}-\left(\varepsilon+\frac{\varepsilon_{1}}{4}+\frac{\kappa_{2}}{2}+\frac{\kappa_{2} \varepsilon}{2}\right)\left\|\nabla v_{t}\right\|^{2} \\
& +\left(\varepsilon^{2} \varepsilon_{1}-\frac{r_{0}}{2}-\frac{r_{0} \varepsilon}{2}-\frac{\beta q \varepsilon}{2}-\frac{3 r_{0}}{8}-\frac{\alpha p \varepsilon}{4}-\frac{\kappa_{2} \varepsilon_{1} \varepsilon}{2}-\frac{\kappa_{2} \gamma_{1}}{2(K-2 \varepsilon)}\right)\|\Delta v\|^{2} \\
& -\left(\frac{r_{0}}{4}+\frac{\kappa_{2} \varepsilon}{2}\right)\|\nabla v\|^{2}-C .
\end{aligned}
$$

Let $n_{1}=\varepsilon_{1}-\frac{r_{0}}{2}-\frac{\beta q}{2}-\frac{\alpha p}{2} ; n_{2}=\varepsilon+\frac{\varepsilon_{1}}{4}+\frac{\kappa_{2}}{2}+\frac{\kappa_{2} \varepsilon}{2}$;

$$
n_{3}=\varepsilon^{2} \varepsilon_{1}-\frac{r_{0}}{2}-\frac{r_{0} \varepsilon}{2}-\frac{\beta q \varepsilon}{2}-\frac{3 r_{0}}{8}-\frac{\alpha p \varepsilon}{4}-\frac{\kappa_{2} \varepsilon_{1} \varepsilon}{2}-\frac{\kappa_{2} \gamma_{1}}{2(K-2 \varepsilon)} ;
$$




$$
n_{4}=\frac{r_{0}}{4}+\frac{\kappa_{2} \varepsilon}{2} .
$$

By using Poincaré's inequality, we get

$$
\Psi_{2}-\kappa_{2} \Phi_{2} \geq\left(\lambda_{1} n_{1}-n_{2}\right)\left\|\nabla v_{t}\right\|^{2}+\left(\lambda_{1} n_{3}-n_{4}\right)\|v\|^{2}-C .
$$

We take proper $\varepsilon, \varepsilon_{1}, \gamma_{1}, \kappa_{2}, \alpha, r_{0}, \alpha$, such that:

$$
\left\{\begin{array}{l}
\lambda_{1} n_{1}-n_{2} \geq 0 \\
\lambda_{1} n_{3}-n_{4} \geq 0
\end{array}\right.
$$

Then

$$
\Psi_{2}-\kappa_{2} \Phi_{2} \geq-C
$$

From the relation (3.53), we can get

$$
\frac{\mathrm{d}}{\mathrm{d} t} \Phi_{2}(t)+\kappa_{2} \Phi_{2}(t) \leq C, t \geq \tau_{0} .
$$

By using Gronwall's inequality, we obtain:

$$
\Phi_{2}(t) \leq \Phi_{2}\left(\tau_{0}\right) \mathrm{e}^{-\kappa_{2}\left(t-\tau_{0}\right)}+\frac{C}{\kappa_{2}}\left(1-\mathrm{e}^{-\kappa_{2}\left(t-\tau_{0}\right)}\right), t \geq \tau_{0} .
$$

Taking $T_{1} \gg \tau_{0}$, such that $\Phi_{2}\left(T_{0}\right) \mathrm{e}^{-\kappa_{2}\left(t-\tau_{0}\right)} \leq 1$, then

$$
\Phi_{2}(t) \leq 1+\frac{C}{\kappa_{2}}, \forall t \geq T_{1}
$$

where

$$
\begin{aligned}
\Phi_{2} & =\frac{1}{2}\left\|\nabla v_{t}\right\|^{2}+\varepsilon \int_{\Omega} \nabla v \nabla v_{t} \mathrm{~d} x+\frac{\varepsilon \varepsilon_{1}}{2}\|\Delta v\|^{2}+\frac{1}{2} \phi\left(\|\nabla u\|^{2}\right)\|\Delta v\|^{2} \\
& \geq \frac{(1-\varepsilon)}{2}\left\|\nabla v_{t}\right\|^{2}+\frac{\left(\lambda_{1} \varepsilon_{1} \varepsilon-\varepsilon\right)}{\lambda_{1}}\|\Delta v\|^{2} \\
& \geq \frac{(1-\varepsilon)}{2}\left\|\nabla v_{t}\right\|^{2}+\frac{\left(\lambda_{1} \varepsilon_{1} \varepsilon-\varepsilon\right)}{\lambda_{1}}\|\Delta v\|^{2}-\frac{C}{\kappa_{2}} .
\end{aligned}
$$

Meanwhile, we once again take proper $\varepsilon, \varepsilon_{1}$, such that:

$$
\left\{\begin{array}{l}
1-\varepsilon>0 \\
\lambda_{1} \varepsilon_{1} \varepsilon-\varepsilon>0 .
\end{array}\right.
$$

So there are $T_{1}>0, R_{1}>0$, which make the following inequalities:

$$
\|A u(t)\| \leq R_{1},\left\|A^{\frac{1}{2}} u_{t}(t)\right\| \leq R_{1}, \forall t \geq T_{1} .
$$

where $R_{1}$ is independent of the initial value $U_{0}$.

Similar to above discussions, there are $T_{2} \gg T_{1}, R_{2}>0$, which make the following inequalities:

$$
\left\|A^{\frac{3}{2}} u_{t}(t)\right\| \leq R_{2},\left\|A u_{t t}(t)\right\| \leq R_{2}, \forall t \geq T_{2} .
$$

where $R_{2}$ is independent of the initial value $U_{0}$.

Using the original Equation (1.1), we obtain 


$$
\begin{aligned}
& A\left(\varepsilon_{1} u_{t}+\phi\left(\left\|A^{\frac{1}{2}} u\right\|^{2}\right) u\right) \\
& =f(x)-u_{t t}-\alpha\left|u_{t}\right|^{p-1} u_{t}-\beta|u|^{q-1} u \in C_{b}\left(\left[T_{2},+\infty\right) ; D(A)\right)
\end{aligned}
$$

Next, using the elliptic property of the operator A, we get:

$$
\begin{aligned}
& \left\|\varepsilon_{1} u_{t}+\phi\left(\left\|A^{\frac{1}{2}} u\right\|^{2}\right) u\right\|_{D\left(A^{2}\right)} \\
& \leq\|A f(x)\|+\left\|A u_{t t}\right\|+\left\|A\left(\alpha\left|u_{t}\right|^{p-1} u_{t}\right)\right\|+\left\|A\left(\beta|u|^{q-1} u\right)\right\| \leq R_{3}, \forall t \geq T_{2}
\end{aligned}
$$

where $R_{3}$ is independent of the initial value $U_{0}$.

So there are $\tau_{1} \gg T_{2}, K_{1}>0$, which make the following inequalities:

$$
\|u(t)\|_{D\left(A^{2}\right)} \leq K_{1},\left\|u_{t}(t)\right\|_{D\left(A^{2}\right)} \leq K_{1}, \forall t \geq \tau_{1} \text {. }
$$

where $K_{1}$ is independent of the initial value $U_{0}$.

According to Lemmas 3.1, 3.2, we can get the following theorem :

Theorem 3.1 From reference [14], let $S(t)$ is the semigroup operator for the problems (1.1) - (1.3), then the semigroup $S(t)$ exists a compact global attractor $\mathcal{A}_{1}$ in $D\left(A^{2}\right)$, and $\mathcal{A}_{1}=\mathcal{A}_{0}$.

The proof of theorem 3.1 see ref. [14], is omitted here.

\section{The Approximate Inertial Manifold for the Global Attractor}

In this section, we first construct a smooth manifold $\mathcal{M}_{1}=\operatorname{graph}\left(\psi_{0}\right)$, and then prove that $\mathcal{M}_{1}$ is an approximate inertial manifold of the semigroup $S(t)$, namely, the arbitrary trajectory of the Kirchhoff wave equations goes into a small neighbourhood of the approximate inertial manifold after large time.

Let $E_{N}=\operatorname{span}\left\{\omega_{k}\right\}_{k=1}^{N}, P_{N}$ is an orthogonal projection from the space $E$ to the subspace spanned by $E_{N}=\operatorname{span}\left\{\omega_{k}\right\}_{k=1}^{N}, Q_{N}=I-P_{N}$, so that $u$ is decomposed as the sum $u=p+q$.

For the solution $u$ of the problems (1.1) - (1.3), let $p=P_{N} u, p_{t}=P_{N} u_{t}, q=Q_{N} u$, $q_{t}=Q_{N} u_{t}$. Then $\xi=\left(p, p_{t}\right)^{\mathrm{T}}, \zeta=\left(q, q_{t}\right)^{\mathrm{T}}, g(u)=|u|^{q-1} u, h\left(u_{t}\right)=\left|u_{t}\right|^{p-1} u_{t}$. We use $P_{N}$ and $Q_{N}$ to act the problem (1.1) respectively.

$$
\begin{aligned}
& p_{t t}+\varepsilon_{1} A p_{t}+\phi\left(\left\|A^{\frac{1}{2}} u\right\|^{2}\right) A p+P_{N}\left(\beta g(p+q)+\alpha h\left(p_{t}+q_{t}\right)\right)=P_{N} f(x), \quad x \in \Omega, \\
& q_{t t}+\varepsilon_{1} A q_{t}+\phi\left(\left\|A^{\frac{1}{2}} u\right\|^{2}\right) A q+Q_{N}\left(\beta g(p+q)+\alpha h\left(p_{t}+q_{t}\right)\right)=Q_{N} f(x), \quad x \in \Omega .
\end{aligned}
$$

Let $\widetilde{P_{N}}=\left(\begin{array}{cc}P_{N} & 0 \\ 0 & P_{N}\end{array}\right), \widetilde{Q_{N}}=\left(\begin{array}{cc}Q_{N} & 0 \\ 0 & Q_{N}\end{array}\right)$. Then the problems (4.63) - (4.64) can be written as:

$$
\xi_{t}+\Lambda \xi=\widetilde{P_{N}} F(\xi+\zeta)
$$




$$
\zeta_{t}+\Lambda \zeta=\widetilde{Q_{N}} F(\xi+\zeta)
$$

From above, we have $\forall U_{0} \in D(A) \times E$, there exist $\tau_{1}, K_{1}>0$, is independent of the initial value $U_{0}$, and then $U(\cdot) \in C_{b}\left(\left[\tau_{1},+\infty\right), D(A) \times E\right)$,

$$
\begin{gathered}
\|u(t)\|_{D\left(A^{2}\right)} \leq K_{1},\left\|u_{t}(t)\right\|_{D\left(A^{2}\right)} \leq K_{1}, \forall t \geq \tau_{1} \text {. So for } q=Q_{N} u, \quad q_{t}=Q_{N} u_{t} \text {, we obtain } \\
\|q\| \leq K_{1} \lambda_{N+1}^{-2},\left\|q_{t}\right\| \leq K_{1} \lambda_{N+1}^{-2}, \forall t \geq \tau_{1} .
\end{gathered}
$$

Theorem 4.1 From references [14] [15] [16], according to lemmas 3.1, 3.2 and the theorem 3.1, let $\mathcal{M}_{0}=\widetilde{P_{N}}(D(A) \times E)$ is the $N$ dimensional linear subspace of $D(A) \times E$, there exists $\tau_{1}>0, \tau_{1}$ is sufficiently large. When $t>\tau_{1}$, arbitrary trajectory arising from the $U_{0}$ for the Kirchhoff wave equations, which track into a $K_{1} \lambda_{N+1}^{-2}$ sphere in $\mathcal{M}_{0}$. Namely, $\operatorname{dist}_{D(A) \times E}\left(S(t) U_{0}, \mathcal{M}_{0}\right) \leq K_{1} \lambda_{N+1}^{-2}$. Meanwhile, the $\mathcal{M}_{0}$ is called a $N$ dimensional flat approximate inertial manifold of the semigroup $S(t)$.

Remark 4.1. For the problem (4.66), if we do not consider $\zeta_{t}$ and $\zeta$ contained in the nonlinear terms, for $\xi \in\left(E_{N}\right)^{2}$, we define mapping $\psi_{0}: \xi \mapsto \psi_{0}(\xi) . \zeta_{0}:=\psi_{0}(\xi)$ is the solution of the Equation (4.68):

$$
\Lambda \zeta_{0}=\widetilde{Q_{N}} F(\xi) .
$$

Then $\psi_{0}:\left(E_{N}\right)^{2} \rightarrow\left(Q_{N} E\right)^{2}$ is a smooth map, its image is $\mathcal{M}_{1}=\operatorname{graph}\left(\psi_{0}\right)=\left\{\xi+\psi_{0}(\xi) \mid \xi \in\left(E_{N}\right)^{2}\right\}$, which is a approximate inertial manifold of the semigroup $S(t)$.

Theorem 4.2 From references [14] [15] [16], according to lemmas 3.1, 3.2 and the theorems 3.1, 4.1, then $\forall U_{0} \in D(A) \times E$, there exists $\tau_{1}>0$, when $t>\tau_{1}$, arbitrary trajectory arising from the $U_{0}$ for the Kirchhoff wave equations, which track into a $K_{1} \lambda_{N+1}^{-1}$ neighborhood in $\mathcal{M}_{1}$. Namely, dist ${ }_{D(A) \times E}\left(S(t) U_{0}, \mathcal{M}_{1}\right) \leq K_{1} \lambda_{N+1}^{-1}$. Meanwhile, the $\mathcal{M}_{1}$ is a approximate inertial manifold of the semigroup $S(t)$. Furthermore, $\forall U_{0} \in D(A) \times E$, there exists $\tau_{n}>0, \tau_{n}$ is sufficiently large, $n \geq 1$. When $t>\tau_{n}$, arbitrary trajectory arising from the $U_{0}$ for the Kirchhoff wave equations, which track into a $K_{n} \lambda_{N+1}^{-n}$ neighborhood in $\mathcal{M}_{n}$. Namely, $\operatorname{dist}_{D(A) \times E}\left(S(t) U_{0}, \mathcal{M}_{n}\right) \leq \widetilde{C_{n}} K_{n} \lambda_{N+1}^{-n}$. The $\mathcal{M}_{n}$ is a very precise approximate inertial manifold of the semigroup $S(t)$.

Proof. Firstly, let $U(t)=S(t) U_{0}$, then $\xi(t)=\left(p(t), p_{t}(t)\right)^{\mathrm{T}}:=\widetilde{P_{N}} U(t)$, $\zeta(t)=\left(q(t), q_{t}(t)\right)^{\mathrm{T}}:=\widetilde{Q_{N}} U(t)$ are the solutions of the problems (4.65) - (4.66), and then let $\zeta_{0}(t)=\left(q_{0}(t), q_{0 t}(t)\right)^{\mathrm{T}}:=\psi_{0}(\xi(t)) . W(t)=(\omega(t), \tilde{\omega}(t))^{\mathrm{T}}$.

From the relation (4.68), we can obtain:

$$
\begin{gathered}
\phi\left(\left\|A^{\frac{1}{2}} u\right\|^{2}\right) A q_{0}=Q_{N}\left(f-\beta g(p)-\alpha h\left(p_{t}\right)\right) \\
q_{0 t}=0 .
\end{gathered}
$$

Then from the hypothesis $\left(\mathrm{G}_{1}\right), \varepsilon_{1} \varepsilon \leq \phi\left(\left\|A^{\frac{1}{2}} u\right\|^{2}\right)$.

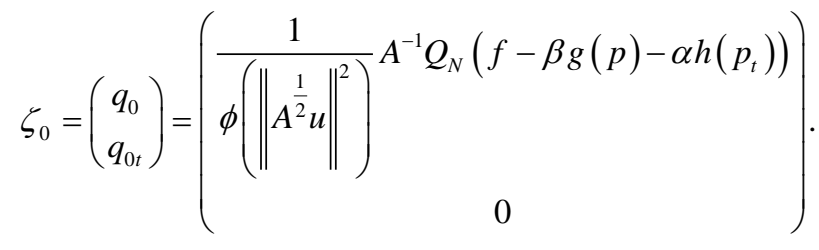




$$
\begin{gathered}
W(t)=(\omega(t), \tilde{\omega}(t))^{\mathrm{T}}=U(t)-\left(\xi(t)+\zeta_{0}(t)\right)=\zeta(t)-\zeta_{0}(t) . \\
\operatorname{dist}_{D(A) \times E}\left(S(t) U_{0}, \mathcal{M}_{1}\right) \leq\|W(t)\|_{D(A) \times E} .
\end{gathered}
$$

We put $W(t)$ into the relation (4.68), the following relations can be obtained immediately,

$$
\begin{gathered}
\phi\left(\left\|A^{\frac{1}{2}} u\right\|^{2}\right) A \omega=\phi\left(\left\|A^{\frac{1}{2}} u\right\|^{2}\right) A q-\phi\left(\left\|A^{\frac{1}{2}} u\right\|^{2}\right) A q_{0} \\
=Q_{N}\left[\left(\beta g(p)+\alpha h\left(p_{t}\right)\right)-\left(\beta g(p+q)+\alpha h\left(p_{t}+q_{t}\right)\right)\right]-q_{t t}-\varepsilon_{1} A q_{t} \\
\tilde{\omega}=q_{t} .
\end{gathered}
$$

Therefore

$$
\begin{gathered}
\phi\left(\left\|A^{\frac{1}{2}} u\right\|^{2}\right)\left\|A^{2} \omega\right\| \leq\left\|A Q_{N}[\beta g(p)-\beta g(p+q)]\right\| \\
+\left\|A Q_{N}\left[\alpha h\left(p_{t}\right)-\alpha h\left(p_{t}+q_{t}\right)\right]\right\|+\left\|A q_{t t}\right\|+\varepsilon_{1}\left\|A^{2} q_{t}\right\| \\
\leq C_{1}\left(\varepsilon, \alpha, \beta, \varepsilon_{1}\right) K_{1}, t \geq \tau_{1} . \\
\|A \tilde{\omega}\| \leq K_{1}, t \geq \tau_{1} .
\end{gathered}
$$

Then

$$
\|A \omega\| \leq C_{1} K_{1} \lambda_{N+1}^{-1},\|\tilde{\omega}\| \leq K_{1} \lambda_{N+1}^{-1}, t \geq \tau_{1} .
$$

So, we obtain

$$
\operatorname{dist}_{D(A) \times E}\left(S(t) U_{0}, \mathcal{M}_{1}\right) \leq\|A \omega\|+\|\tilde{\omega}\| \leq\left(C_{1}+1\right) K_{1} \lambda_{N+1}^{-1}:=\widetilde{C}_{1} K_{1} \lambda_{N+1}^{-1}, t \geq \tau_{1} .
$$

A similar method in reference [14], we immediately get the semigroup $S(t)$ exists a compact global attractor $\mathcal{A}_{n}$ in $D\left(A^{n}\right)$, and $\mathcal{A}_{0}=\mathcal{A}_{1}=\cdots=\mathcal{A}_{n}$, and then $\forall U_{0} \in D(A) \times E$, there exists $\tau_{n}>0, \tau_{n}$ is sufficiently large, $n \geq 1$. When $t>\tau_{n}$, arbitrary trajectory arising from the $U_{0}$ for the Kirchhoff wave equations, which track into a $K_{n} \lambda_{N+1}^{-n}$ neighborhood in $\mathcal{M}_{n}$.

$$
\operatorname{dist}_{D(A) \times E}\left(S(t) U_{0}, \mathcal{M}_{n}\right) \leq \widetilde{C_{n}} K_{n} \lambda_{N+1}^{-n} .
$$

where the $\mathcal{M}_{n}$ is a smooth manifold that we construct, which is very precise, to approximate inertial manifold of the semigroup $S(t)$.

Remark 4.2. This article is based on the references [14] [15] [16], by estimating the higher regularity of the global attractor, then we construct its approximate inertial manifold. Approximate inertial manifold, which is a kind of nonlinear, finite dimensional and has certain smoothness. It is of great significance to study the long time behavior of the dissipative equations and the structure of the attractors. On the basis of this article, then we are likely to consider the inertial manifold of the global attractor for the problems (1.1) - (1.3).

\section{Acknowledgements}

The authors express their sincere thanks to the anonymous reviewer for his/her careful 
reading of the paper, giving valuable comments and suggestions. These contributions greatly improved the paper. This work is supported by the Nature Science Foundation of China (No. 11561076).

\section{References}

[1] Foias, G., Sell, G.R. and Teman, R. (1985) Varities Inertilles des Equations Differentielles Dissipatives. Comptes Rendus de 1 Académie des Sciences, 301, 139-142.

[2] Margolin, L.G. and Jones, D.A. (1992) An Approximate Inertial Manifold for Computing Burgers' Equation. Physica D, 60, 175-184. https://doi.org/10.1016/0167-2789(92)90234-E

[3] Chueshov, I.D. (1996) On a Construction of Approximate Inertial Manifolds for Second Order in Time Evolution Equations. Nonlinear Analysis, Theory, Methods and Applications, 26, 1007-1021. https://doi.org/10.1016/0362-546X(94)00191-4

[4] Jolly, M.S., Kevrekidis, I.G. and Titi, E.S. (1990) Approximate Inertial Manifolds for the Kuramoto-Sivashinsky Equation: Analysis and Computations. Physica D, 44, 38-60. https://doi.org/10.1016/0167-2789(90)90046-R

[5] Babin, A.V. and Vishik, M.I. (1992) Attractors of Evolution Equations. Studies in Mathematics and Its Applications, 25, North-Holland, New York.

[6] Lin, G.G. (2011) Nonlinear Evolution Equation. Yunnan University Press, Kunming.

[7] Dai, Z.D. and Guo, B.L. (2000) Inertial Manifold and Approximate Inertial Manifold. Science Press.

[8] Kirchhoff, G. (1883) Vorlesungen Uber Mechanik. Teubner, Leipzig.

[9] Nakao, M. (2009) An Attractor for a Nonlinear Dissipative Wave Equation of Kirchhoff Type. Journal of Mathematical Analysis and Applications, 353, 652-659. https://doi.org/10.1016/j.jmaa.2008.09.010

[10] Yang, Z.J., Ding, P.Y. and Liu, Z.M. (2014) Global Attractor for the Kirchhoff Type Equations with Strong Nonlinear Damping and Supercritical Nonlinearity. Applied Mathematics Letters, 33, 12-17. https://doi.org/10.1016/j.aml.2014.02.014

[11] Hirosawa, F. (2015) A Class of Non-Analytic Functions for the Global Solvability of Kirchhoff Equation. Nonlinear Analysis, 116, 37-63. https://doi.org/10.1016/j.na.2014.12.016

[12] Ai, C.F., Zhu, H.X. and Lin, G.G. (2015) The Global Attractors and Dimensions Estimation for the Kirchhoff Type Wave Equations with Nonlinear Strongly Damped Terms. Journal of Advances in Mathematics, 12, 6087-6102.

[13] Dai, Z.D., Guo, B.L. and Lin, G.G. (1998) The Fractal Structure of Attractor for the Generalized Kuramoto-Sivashinsky Equations. Applied Mathematics and Mechanics, 19, 243-256.

[14] Li, Y.S. and Zhang, W.G. (2000) Regularity and Approximate of the Attractor for the Strongly Damped Wave Equation. Acta Mathematica Scientia, 20, 342-350.

[15] Luo, H., Pu, Z.L. and Chen, G.G. (2002) Regularity of the Attractor and Approximate Inertial Manifold for Strongly Damped Nonlinear Wave Equations. Journal of Sichuan Normal University (Natural Science), 25, 459-463.

[16] Wang, L., Dang, J. and Lin, G. (2009) The Approximate Inertial Manifolds of the Fractional Nonlinear Schrodinger Equation. Journal of Yunnan University, 31, 373-377.

[17] Zhang, S. and Zhang, J. (2015) Approximate Inertial Manifold of Strongly Damped Wave Equation. Pure Mathematics, 5, 278-283. https://doi.org/10.12677/PM.2015.56040

[18] Tian, L. and Lin, Y. (1999) Approximate Inertial Manifolds by Spline Wavelet Basis in Weakly Damped Forced KdV Equation. Acta Mathematica Scientia, 19, 379-386. 
[19] Shang, Y. and Guo, B. (1999) Approximate Inertial Manifolds for the Nonlinear Sobolev-Galpern Equations. Acta Mathematica Scientia, 24, 105-115.

[20] Debussche, A. and Marion, M. (1992) On the Construction of Families of Approximate Inertial Manifolds. Journal of Differentital Equations, 100, 173-201.

https://doi.org/10.1016/0022-0396(92)90131-6

[21] Li, Y., Wang, B. and Yang, B. (1997) Regularity and Approximate Inertial Manifolds for a Class of Evolutionary Equations. Journal of Lanzhou University, 33, 10-16.

[22] Showwalter, R.E. (1976) Regularization and Approximation of Second Order Evolution Equations. SIAM Journal on Mathematical Analysis, 7, 461-472. https://doi.org/10.1137/0507037

[23] Li, H., Pu, Z. and Chen, G. (2008) Approximate Inertial Manifolds for the Suspension Bridge Equations. Journal of Sichuan Normal University, 31, 25-30.

[24] Guo, B. and Lin, G. (1999) Approximate Inertial Manifolds of Non-Newtonian Viscous Incompressible Fluids. Journal of Mathematical Study, 32, 328-340.

[25] Teman, R. (1998) Infinite Dimensional Dynamics Systems in Mechanics and Physics. Springer, New York.

[26] Massat, P. (1983) Limiting Behavior for Strongly Damped Nonlinear Wave Equations. Journal of Differentital Equations, 48, 334-349. https://doi.org/10.1016/0022-0396(83)90098-0

[27] Pazy, A. (1983) Semigroup of Linear Operators and Applications to Partial Differential Equations. Springer, Berlin. https://doi.org/10.1007/978-1-4612-5561-1

\section{Submit or recommend next manuscript to SCIRP and we will provide best service} for you:

Accepting pre-submission inquiries through Email, Facebook, LinkedIn, Twitter, etc.

A wide selection of journals (inclusive of 9 subjects, more than 200 journals)

Providing 24-hour high-quality service

User-friendly online submission system

Fair and swift peer-review system

Efficient typesetting and proofreading procedure

Display of the result of downloads and visits, as well as the number of cited articles

Maximum dissemination of your research work

Submit your manuscript at: http://papersubmission.scirp.org/

Or contactijmnta@scirp.org 